\author{
Biagio Moretti \\ Raffaele Garofalo \\ Stefania Genco \\ Vittorio Patella \\ Elyazid Mouhsine
}

\section{Medium-energy shock wave therapy in the treatment of rotator cuff calcifying tendinitis}

Received: 31 March 2004

Accepted: 25 November 2004

Published online: 30 March 2005

(c) Springer-Verlag 2005
B. Moretti $\cdot$ S. Genco $\cdot$ V. Patella Department of Orthopaedics, University of Bari, Bari, Italy

R. Garofalo · E. Mouhsine $(\square)$ Orthopaedic and Traumatology Unit, University of Lausanne, OTR-BH 14, CHUV, 1011 Lausanne, Switzerland E-mail: Elyazid.Mouhsine@hospvd.ch Tel.: +41-21-3142791

Fax: + 41-21-3142800

\begin{abstract}
To evaluate the results of the treatment with medium-energy extracorporeal shock wave therapy (ESWT) in rotator cuff calcifying tendinitis. Fifty-four non-consecutive patients, who were referred to our institute for rotator cuff calcifying tendinitis, were managed with a standardized protocol in four sessions of medium-energy $(0.11 \mathrm{~mJ} /$ $\mathrm{mm}^{2}$ ) ESWT administered with an electromagnetic lithotriptor. Pain was evaluated at the end of each session, functional state of shoulder was assessed at 1 and 6 months after the end of procedure. All patients underwent radiographs and sonography imaging. No systemic or local complications. Thirty-eight patients
\end{abstract}

$(70 \%)$ reported satisfactory functional results. Radiographs and sonographs showed a disappearance of calcium deposit in 29 patients $(54 \%)$ and in 19 patients $(35 \%)$ it appeared to be reduced more than a half. A correlation was found between residual calcium deposit and the clinical outcome, but some patients showed a reduced pain without modification of calcium deposit. These results were unmodified at 6 months follow-up. Our protocol of medium-energy ESWT provides good results overall about pain modulation.

Keywords Rotator cuff Calcification $\cdot$ Shock wave

\section{Introduction}

Rotator cuff calcifying tendinitis is a common disorder with controversial aetiology. Two different processes leading to calcium deposit have been proposed: degenerative calcification [5] and reactive calcification on a healthy tendon [31]. Codman [5] proposed that tendon fibre degeneration precedes calcification and, according to Moseley [22], he defined the tendon-bone insertion area as "critical zone".

Clinically, it's very important to distinguish calcifying tendinitis from a rotator cuff tear as a source of shoulder pain, because the treatment is different.

The treatment of calcifying tendinitis is typically conservative, including use of systemic non-steroidal anti-inflammatory drugs (NSAIDs), physical therapy such as ultrasound, laser or local x-ray, intrabursal injections of corticosteroid and lavage or multiple perforations of deposits using large-bore needles [31]. Distinguishing between the formative and resorptive phase [31] is very important to choose the more appropriate means of conservative treatment. Nevertheless, these different treatments have failed to show reproducible and reliable results $[26,31]$.

Topical steroid injections are usually effective, but often frequent injections are required that are harmful to tendon structure. Percutaneous needle aspiration with a large-size needle $(>n 18)$ alleviates symptoms in up to $60 \%$ of patients [10]; however, larger needle size may cause tendon injury and so acute inflammation. Wittenberg et al. [33] has found that conservative management for calcifying tendinitis leads to less favourable 
pain results in the long term than surgical treatment. For patients with a chronic calcifying tendonitis, surgical removal of the deposits, either with an open procedure or endoscopically, has been reported to relieve symptoms once that conservative therapy fails $[1,21]$. Radiation therapy was ineffective, as reported by Plenk [24]. There are many reports about use of extracorporeal shock wave therapy (ESWT) in musculoskeletal disorders such as pseudarthrosis [27], non-union [29], or in soft tissue pathology, such as plantar fascitiis [4, 23], and elbow epicondylitis [17].

Loew et al. [18] and Rompe et al. [25], independently, reported the first experience about use of ESWT in treatment of shoulder calcifying tendinitis showing encouraging results. The rationale was to disrupt calcific intratendinous deposit in order to encourage its resorption through an increase of local vascularization reducing the shoulder pain. ESWT are classified according to their energy levels. Low energy ESWT have a focal energy flux density (EFD) of up to $0.08 \mathrm{mj} / \mathrm{mm}^{2}$, medium-energy an EFD between $0.09 \mathrm{mj} / \mathrm{mm}^{2}$ and $0.28 \mathrm{mj} / \mathrm{mm}^{2}$ and high-energy up to $0.6 \mathrm{mj} / \mathrm{mm}^{2}$.

There is still no consensus on appropriate doses of ESWT to treat calcifying tendinitis of shoulder, and the doses reported in different reports were operatordependent $[11,14,18,25]$. In the literature, high-dose seems to be associated with good results, but a potential for local soft-tissue damage and important pain is reported [19].

The aim of this study was to evaluate the effectiveness of medium-energy ESWT to treat chronic rotator calcifying tendinitis, and to verify the association with soft tissue damage and pain.

\section{Materials and methods}

Between 10 January and 17 December 2001, 54 nonconsecutive patients suffering from calcifying tendinitis were treated with ESWT in our department.

There were 20 men and 34 women with a mean age of 43 years (range 34-66). There were 32 right and 22 left shoulders, and dominant arm was involved in 34 cases $(63 \%)$.

The inclusion criteria were: the presence of persistent shoulder pain, refractory to conservative treatment, provided for at least three months, including NSAIDs, at least one corticosteroid injection, physical therapy and immobilisation of shoulder in a sling during the acute phase of pathology.

At least 6 weeks before ESWT was done, the abovementioned therapies were discontinued. Absence of contraindication for shock wave treatment, such as recent trauma and local infection, cardiac arhythmia, pacemaker implant, coagulopathy, pregnancy and patients with massive cuff rotator tears, was studied.
All patients underwent clinical examination that was performed by the same author; the Constant score [6] and pain were calculated.The intensity of pain was calculated with the visual analogue scale (VAS) from 0 to 10 points, where 0 represents the absence of pain and 10 unbearable pain.

Radiographs in true anteroposterior (AP) of shoulder, acromial outlet view and sonography were evaluated by the first author to study the type of calcium deposit according to DePalma criteria [9].

Patients who suffered from rotator cuff tears were not included in the study. Treatment was performed in outpatients.All subjects underwent ESWT complaining of chronic symptoms and in no case acute subacromial bursitis was revealed.

Medium-energy ESWT was administered with an electromagnetic lithotriptor, Minilith SL1 (Karl Storz Medical, Tuttlingen, Germany), which presents a C-arm adjustable fluoroscopic unit. All patients who underwent treatment presented a type II deposit according to DePalma [9]. Each patient received 2500 pulses of shock waves $\left(0.11 \mathrm{~mJ} / \mathrm{mm}^{2}\right.$ EFD) per session, as reported by some authors [12, 28]; 120 impulses per minute were applied. Our protocol of treatment was provided in four sessions at 3 days interval. All therapy was carried-out without anaesthesia. No cold therapy or NSAIDs was allowed after the procedure.

\section{Method of evaluation}

The patients were instructed to complete a pain scale (VAS) after each session, to report any reddening of the skin, swelling, petechiae, bleeding, acute bursitis, or syncope. The patients were assessed, after a follow-up period of 1 and 6 months by the same author who evaluated them before treatment. At follow-up, assessment was carried-out using the Constant score and VAS. The resumption and level of working activity were investigated. An AP X-ray of the affected shoulder, acromial outlet view and sonography were evaluated always by the first author to study the evolution of calcification.

The assessement of calcium deposit size was performed at the X-rays. In particular the films obtained at 1 month and 6 months were to place on the X-ray obtained at the time zero to evaluate differences about the calcium deposit size. To eliminate any problems related to X-ray device and technician, the technique was standardized in terms of position of shoulder and arm,distance from the radiographic film, exposure [16], and team of technicians.

The outcome was considered to be excellent if there was a reduction of pain between the $91 \%$ and $100 \%$, a resumption of working activity without limitations, and 
Constant score between 86 and 100 points. The outcome was considered as good, if the reduction of pain was between $71 \%$ and $90 \%$, working activity was resumed with mild limitation, and Constant score was between 66 and 85 points. The outcome was considered fair if there was a reduction of pain between $41 \%$ and $70 \%$, a resumption of working activity with moderate limitations, and Constant score between 51 and 65 points. The cases were considered poor when a reduction of pain was not referred, resumption of working activity with severe limitation, and Constant score between 25 and 50 points. Statistical analysis was performed using nonparametric Wilcoxon test for two independent samples. Statistical significance was set at a $P$ value of $<0.05$.

\section{Results}

No patient, but one, in which a local reddening was reported, referred systemic or local complications. The curve of mean values of pain reported by patients after each session of treatment is reported on Fig. 1.

Using our criteria of evaluation, at the last follow-up, 24 patients $(44 \%)$ were considered to be excellent, 14 $(26 \%)$ good, $12(23 \%)$ fair and $4(7 \%)$ poor. Working activity was resumed at a mean time of 12 days (range 10-18) from last therapy. The mean Constant score at 1month follow-up was 68.2 (42-92), versus 24.5 (16-44) before treatment. The differences between these values were significant $(P=0.002)$.

The mean pain-score was 4.5 (3-7) before treatment and $1.92(0-5)$ at follow-up $(P=0.03)$. In particular, only the four patients $(7.4 \%)$ who were classified as poor complained of unmodified pain, overall during the activity (VAS:5/10). Six other patients $(12 \%)$ complained of occasional pain (VAS:2/10). None of the patients reported a worsening of symptoms.

These data remained unvaried after 6-month followup. At the last follow-up there were differences in the Constant score between treated shoulder and contralateral side, respectively 68.2 (42-93) and 86 (78-98), but not significant $(P=0.07)$.

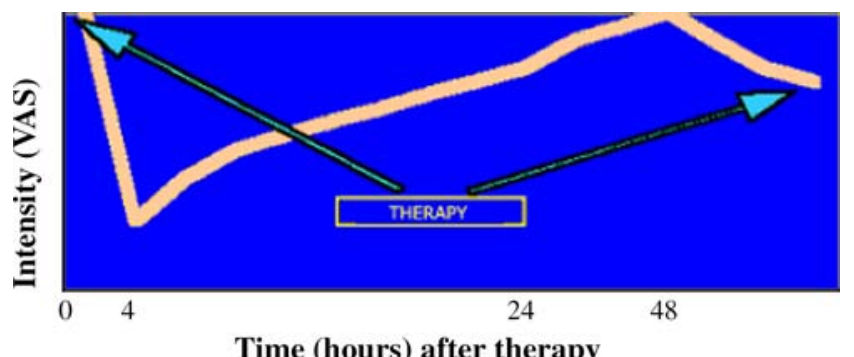

Fig. 1 Typical development of pain according to VAS (range 0-10) at baseline and after medium-energy ESWT
Radiographic and ultrasonographic evaluation, showed that at 1 month the calcium deposit had disappeared in 29 patients $(54 \%)$ and in 19 patients $(35 \%)$ it appeared reduced by more than half. In the remaining 6 patients $(11 \%)$, calcium deposit appeared unchanged. These findings appeared unvaried at 6 months of followup (Figs. 2, 3).

Evaluating these results, a correlation seems to exist between the residual volume of calcium deposit and the clinical outcome. In fact, of the 6 patients in whom calcium deposit appeared unchanged, four presented poor results, and the other two showed a fair result.

\section{Discussion}

Calcifying tendinitis of rotator cuff is an important source of shoulder pain with a contradictory natural history $[2,5,31]$.

Bosworth [3] described, in a prospective study, that within 3 years after the diagnosis, the calcific deposit disappeared in $9.3 \%$ of patients, but acute symptoms can recur when the calcification remains more than $15 \mathrm{~mm}$ in diameter. Investigations at 10-year follow-up have found a disappearance of deposit in $27.1 \%$ of patients [32].

A limited response to most conservative treatments has led to the search for new forms of treatment. Shock waves have demonstrated the capability of stone disintegration, presumably through high stress forces on the stone surface by the high-pressure amplitude and the short rise time, thus exceeding the elastic strength of the stone and disintegrating its surface [23]. At the same time, increased vascularization in the area of treated

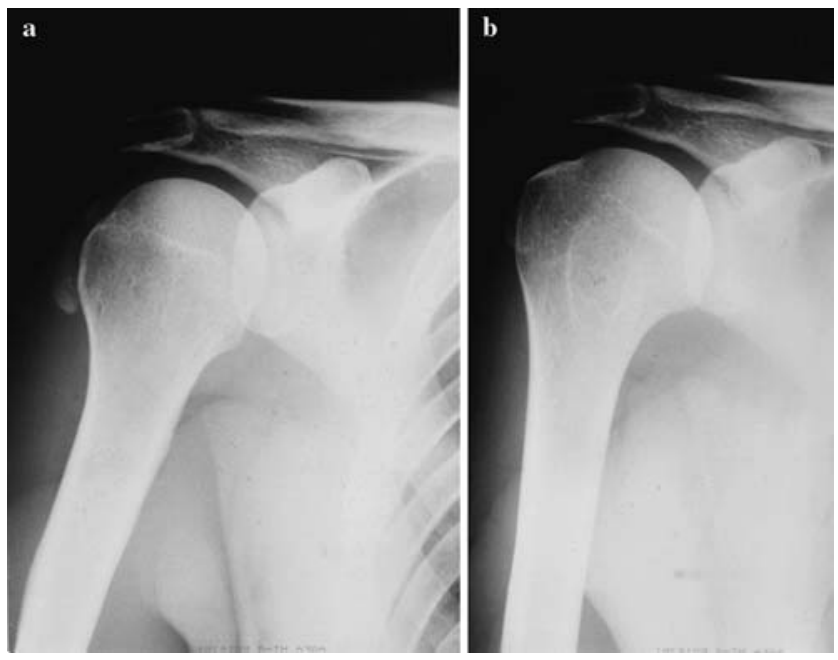

Fig. 2 a AP X-ray views of patient with De Palma Type II calcium deposit. b AP X-ray showing complete disappearance 6 months after ESWT 


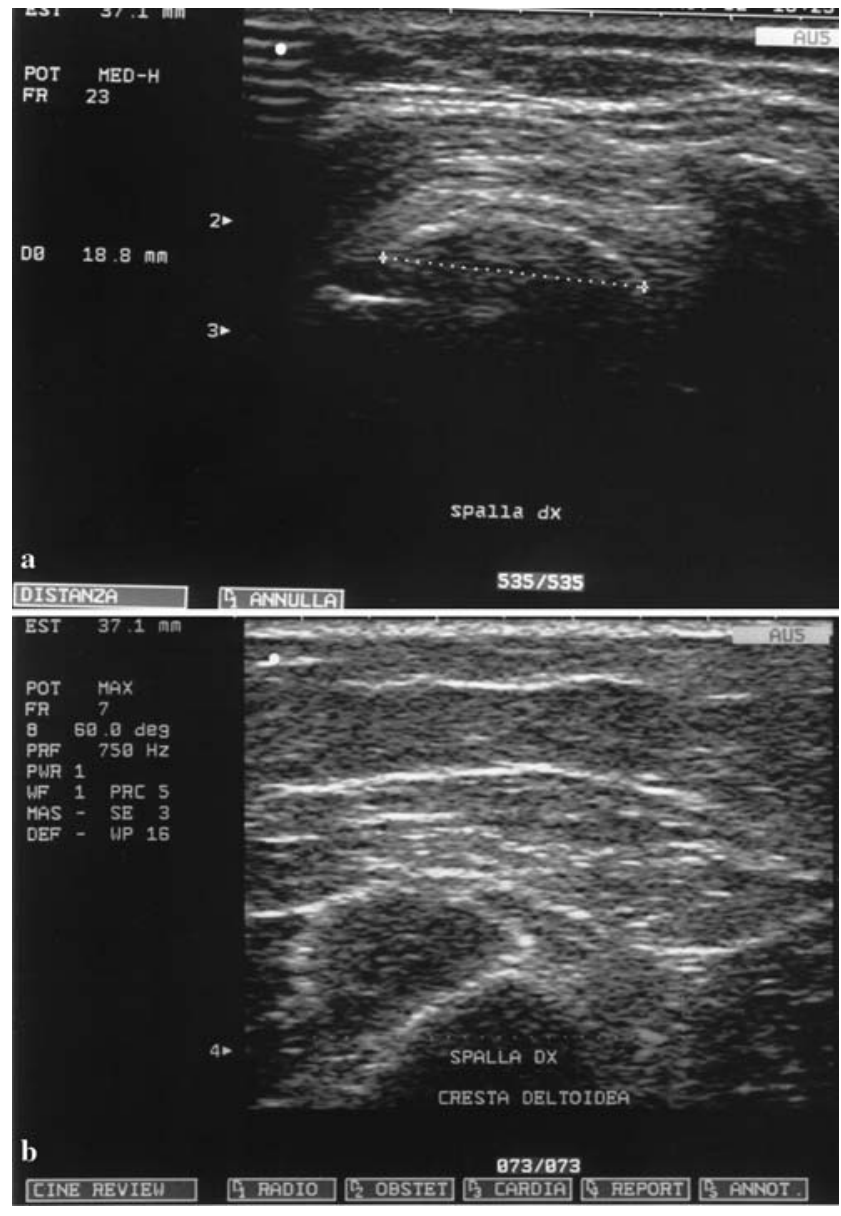

Fig. 3 a Transverse sonographic image of right supraspinatus tendon showing arc type calcific plaque before ESWT. b Sonographic control at 6 months showing disappearance of deposit

tendon appears to lead to accelerated breakdown of the remaining calcific structure.

The goal in the treatment of rotator cuff calcifying tendinitis is the disappearance of calcium deposit and reduction of pain.

The present study demonstrated the effectiveness of medium-energy ESWT in the treatment of calcifying tendinitis of shoulder. At last follow-up, at six months, $70 \%$ of the patients presented a satisfactory functional result. About 23\% reported a little subjective and objective recovery, and only 4 patients $(7 \%)$, classified as poor results, complained of activity-related pain. In these patients, the calcium deposit at follow-up appeared unchanged. In the series, in fact, only in $11 \%$ of patients it appeared unmodified, and, as discussed, a correlation between disappearance of deposit and clinical outcome seems to exist, according to other authors [18].

The complete disappearance of the calcium deposit was noted in $54 \%$ of the patients. This result is similar to data reported by Gerdesmeyer et al. [11], which found a disapparition of calcium deposit in $60 \%$ of the patients who were treated with high-energy ESWT.

Our clinical results agree with the results reported in other series [7, 18, 25, 26, 29], but in our series no differences were found between results at 1 month and 6 month follow-up.

The effectiveness of high-energy ESWT to relieve the pain of patients with calcifiyng tendinitis has been shown by different authors [11, 14, 18]. Jakobeit et al. [14] in particular reported $85 \%$ satisfactory results.

Gerdesmeyer et al. [11] have recently showed that both high-energy and low-energy ESWT appeared to provide a beneficial effect in case of calcifying tendinitis of rotator cuff, but high-energy ESWT was superior to low-energy ESWT. These outcomes are similar to results reported in the open [31] and arthroscopy [1, 15, 21] surgery series.

A study carried-out by Rompe et al. [26], evaluating ESWT versus conventional surgery, have shown little differences about clinical outcome. Patients treated with ESWT returned to work at a mean time of 15 days, 12 days in our series, as compared to 9 weeks reported in patients treated with surgery [26].

Our results are similar to those of other series in which high-energy ESWT in more than one session was carried-out [7, 11, 14, 19], and better compared to papers where the use of high-energy ESWT [26] or mediumenergy ESWT [30] in a single session was reported. In any case, the results are worse, compared to surgical treatment [26].

It is very interesting to note the curve of pain (Fig.1) after treatment with ESWT; immediately after each session of treatment and 4-5 hours following, we have noted a reduction of pain, whereas during following 24 48 hours the pain increased at a level, in any case, inferior with respect to the initial one.

The intensity of pain was always lower in the following sessions. This effect seems to be related to inhibition of pain receptors or denervation [13].

Some studies have found that shock wave seems to distort axonal contents, increasing the permeability and leading to depolarization; so the nociceptors loose their ability for generation of potentials necessary to elicit the pain signal response [23]. The pain relief, moreover, seems to be a function of the total applied energy [23].

These findings support our protocol of treatment, four sessions of medium-energy ESWT, and can explain why some patients, with calcium deposit persistence, referred amelioration of symptoms. Nevertheless, the response is very individual.

A last point concerns the side-effects of ESWT. In our series, we don't use local anaesthesia or intravenous analgesia during treatment, and the patients were able to tolerate the pain. In the series in which the high-energy was used (gerdesm), the treatment was carried-out under intravenous analgesia. Moreover, the potential for 
haemorrage and local tissue damage is well recognized with the use of ESWT. In our series, only one patient $(5 \%)$ complained of a local reddening during therapy. This percentage is lower than that reported in treatment using high-energy ESWT [11], and confirm the findings of Delius [8], who found that soft tissue damage and side effects are more likely with high-dose ESWT.

So far, to our knowledge, this protocol of treatment has not been analysed previously in a clinical setting.

The main quality of our study was the way of recruitment of patients evaluated clinically by only one author, as also the radiographic and sonographic examinations, to be sure that the patients presented a chronic phase of disease. Maier et al. [20] performing a pre-treatment MRI study, found the best outcomes after ESWT, in patients with chronic tendinitis.

Anyway the follow-up, in our study, can be considered a good one, but it was chosen to differentiate between the effects of treatment and the natural history of the disease.

The authors think that the medium-energy ESWT is effective for patients suffering from calcifying tendinitis of the shoulder and overall pain modulation, thus allowing daily activity.

In future, prospective multicentric studies should be necessary to define a standard protocol of treatement.

\section{References}

1. Ark JW, Flock TJ, Flatow EL, Bigliani LU (1992) Arthroscopic treatment of calcific tendinitis of the shoulder. Arthroscopy 8:183-188

2. Booth RE, Marvel JP (1975) Differential diagnosis of the shoulder pain. Orthop Clin North Am 6:353-379

3. Bosworth BM (1941) Calcium deposits in the shoulder and subacromial bursitis. JAMA 116:2482-2485

4. Chen HS, Chen LM, Huang TW (2001) Treatment of painful heel syndrome with shock waves. Clin Orthop 387:4146

5. Codman EA (1906) On stiff and painful shoulders. Boston Med Surg J 154:613620

6. Constant CR, Murley AHG (1987) A clinical method of functional assessment of the shoulder. Clin Orthop 214:160164

7. Daecke W, Kusnierczak D, Loew M (2002) Extracorporeal shock wave therapy (ESWT) in tendinosis calcarea of the rotator cuff. Long-term results and efficacy. Orthopade 31(7):645-651

8. De Palma AF, Kruper JS (1961) Long term study of shoulder joints afflicted with and treatment for calcifyng tendinitis. Clin Orthop 20:61-72

9. Delius M (1994) Medical applications and bioeffects of extracorporeal shock waves. Shock Waves 4:55-72

10. Farin PU, Rasanen H, Jaroma H, Jarju (1996) Rotator cuff calcifications: treatment with ultrasound-guided percutaneous needle aspiration and lavage. Skeletal Radiol 25:551-554

11. Gerdersmeier L, Wagenpfeil S, Haake $M$ et al (2003) Extracorporeal shock wave therapy for the treatment of chronic calcifzing tendonitis of the rotator cuff. JAMA 290(19):2573-2580
12. Gimblett PA, Saville J, Ebrall P (1999) A conservative management protocol for calcifyng tendinitis of the shoulder. $\mathbf{J}$ Manipulative Physiol Ther 22(9):622627

13. Haupt G (1997) Use of extracorporeal waves in the treatment of pseudoarthrosis, tendinopathy and other orthopedic diseases. J Urol 158:4-11

14. Jakobeit C, Winiarski B, Jakobeit S, Welp L, Spelsberg G (2002) Ultrasound-guided, high-energy extracorporeal shock wave treatment of symptomatic calcareous tendinopathy of the shoulder. ANZ J Surg 72(7):496500

15. Jerosch J, Strauss JM, Schmiel S (1998) Arthroscopic treatment of calcific tendinitis of the shoulder. J Shoulder Elbow Surg 7:30-37

16. Kilcoyne RF, Reddy PK, Lyons F, Rockwood CA Jr (1989) Optimal plain film imaging of the shoulder impingement syndrome. AJR Am J Roentgenol 153:795-797

17. Ko JY, Chen HS, Chen LM (2001) Treatment of lateral epicondylitis of the elbow with shock waves. Clin Orthop 387:60-67

18. Loew M, Jurgowski W, Mau HC, Thomsen M (1995) Treatment of calcifying tendinitis of rotator cuff by extracorporeal shock waves: a preliminary report. J Shoulder Elbow Surg 4:101-106

19. Loew M, Daecke W, Kusnierczak D, Rahamanzadeh M, Ewerebeck V (1999) Extracorporeal shock wave application: an effective treatment for patients with chronic and therapy-resistant calcifying tendinitis? J Bone Joint Surg 81B:863867
20. Maier M, Steinborn M, Schmitz C, Stabler A, Kohler S, Pfahler M, Durr HR, Refior HJ (2000) Shock wave application in calcifying tendinitis of the shoulder. Prediction of outcome by imaging. Arch Orthop Trauma Surg 120:43-48

21. Molé D, Kempf JF, Gleyze P (1993) Résultats du traitement arthroscopique des tendinopathies non-rompues de la coiffe des rotateurs: 2. Calcifications de la coiffe. Rev Chir Orthop Reparatrice Appar Mot 79:532-541

22. Moseley HF, Goldie I (1963) The arterial pattern of the rotator cuff of the shoulder. J Bone Joint Surg 45-B:780789

23. Ogden JA, Alvarez RG, Levitt R, Marlow M (2001) Shock wave therapy (orthotripsy) in musculoskeletal disorders. Clin Orthop 387:22-40

24. Plenk H (1952) Calcifying tendinitis of the shoulder: a critical study of the value of x-ray therapy. Radiology 59:384 389

25. Rompe JD, Rumler F, Hoph C et al (1995) Extracorporeal shock wave therapy for calcifying tendinitis of the shoulder. Clin Orthop 321:196-201

26. Rompe JD, Zoellner J, Nafe B (2001) Shock wave therapy versus conventional surgery in the treatment of calcifying tendinitis of the shoulder. Clin Orthop 387:72-82

27. Schleberger R, Senge (1992) Noninvasive treatment of long bone pseudoarthrosis by shock wave. Acta Orthop Trauma Surg 111:224-227

28. Seil R, Rupp S, Hammer DS, Ensslin S, Gebhardt T, Kohn DR (1999) Extracorporeal shock wave therapy in tendinosis calcrea of the rotator cuff: comparison of different treatment protocols. Z Orthop Ihre Grenzgeb 137(4):310-314 
29. Shaden W, Fischer A, Sailler A (2001) Extracorporeal chock wave therapy of nonunion or delayed osseous union. Clin Orthop 387:90-94

30. Thiel M (2001) Application of shock waves in medicine. Clin Orthop 387:18 21
31. Uhthoff HK, Loehr JW (1997) Calcific tendinopathy of the rotator cuff. J Am Acad Orthop Surg 5(4):183-191

32. Waghenhäuser JF (1972) Die Periarthropatie-Syndrome. Therapiewoche 37:3187-3192
33. Wittenberg RH, Rubenthaler F, Wolk T, Ludwig J, Willburger RE, Steffen R (2001) Surgical or conservative treatment for chronic rotator cuff calcifying tendinitis-a matched-pair analysis of 100 patients. Arch Orthop Trauma Surg 121:56-59 